Pacific

Journal of

Mathematics

ISOPERIMETRIC INEQUALITIES FOR SECTORS ON SURFACES

HyOUNGSick BAHN AND Sungpyo Hong 


\title{
ISOPERIMETRIC INEQUALITIES FOR SECTORS ON SURFACES
}

\author{
Hyoungsick Bahn And Sungpyo Hong
}

\begin{abstract}
We discuss sectors on a surface of curvature bounded above by a constant and derive an isoperimetric inequality for a proper sector on such a surface. With this isoperimetric inequality we derive an inequality involving the total length of the cut locus of a point on a closed surface.
\end{abstract}

\section{Introduction.}

There have been extensive studies on isoperimetric inequalities on a Riemannian manifold of dimension 2 (shortly, a surface) [Al, BdC, CF, Fi]. C. Bandle derived an isoperimetric inequality for a sector in the Euclidean plane $\mathbb{E}^{2}[\mathbf{B a 1}, \mathbf{B a 2}]$ : Let $D$ be a sector in $\mathbb{E}^{2}$, which is a simply connected region enclosed by two line segments $\gamma_{1}, \gamma_{2}$ starting at a point $p$ and a piecewise smooth simple curve segment $\Gamma$ joining the end points of $\gamma_{1}, \gamma_{2}$. Let $\theta_{0}$ denote the interior angle of $D$ at $p$. C. Bandle showed that for a sector $D$ with $\theta_{0} \leq \pi$,

$$
L^{2}(\Gamma) \geq 2 \theta_{0} A(D)
$$

with equality if and only if $D$ is a circular sector, where $L(\Gamma)$ denotes the length of $\Gamma$ and $A(D)$ the area of $D$.

In this paper, we consider an isoperimetric inequality for a sector on a surface $M$ with curvature $K$ bounded above by a constant $C$. By a sector on a surface $M$ we mean a region of $M$ enclosed by two geodesic segments $\gamma_{1}, \gamma_{2}$ and a piecewise smooth curve segment $\Gamma$, which together constitute a simple closed curve $\Gamma^{*}=\gamma_{1} \cup \Gamma \cup \gamma_{2}$. On a general surface $M$, a sector needs not be simply connected nor bounded (e.g., the cylinder $\mathbb{R} \times \mathbb{S}^{1}$ ), or could be the whole surface (e.g., the torus $T^{2}$ ). On the other hand, such a simple closed curve $\Gamma^{*}$ may enclose two bounded sectors (e.g., the sphere $\mathbb{S}^{2}$ ). For our purpose, we will restrict our attention to sectors on a surface $M$ that are closed, simply connected and bounded ones. We will call such a sector by a proper sector, denoted by $D(\Gamma)$ or just $D$. We take parametrizations of two geodesic segments $\gamma_{i}$ and a piecewise smooth curve segment $\Gamma$ as $\gamma_{i}:\left[0, r_{i}\right] \rightarrow M(i=1,2)$ and $\Gamma:[a, b] \rightarrow M$ such that $\gamma_{1}(0)=\gamma_{2}(0)$ and $\Gamma(a)=\gamma_{1}\left(r_{1}\right), \Gamma(b)=\gamma_{2}\left(r_{2}\right)$ so that $\Gamma^{*}=\gamma_{1} \cup \Gamma \cup \gamma_{2}$ is a simple closed curve with a suitable orientation. The vertex of $D$ is the point $\gamma_{1}(0)=\gamma_{2}(0)$ where $\gamma_{1}$ and $\gamma_{2}$ cross. Our main result is the following: 
Isoperimetric Inequality for a Sector. Let $M$ be a surface with curvature $K$ bounded above by a constant $C$. Let $D$ be a proper sector on $M$ with interior angle $\theta_{0} \leq \pi$ at the vertex. Then

$$
L^{2}(\Gamma) \geq 2 \theta_{0} A(D)-C A^{2}(D) .
$$

Equality holds only when $D$ is isometric to a geodesic sector on a surface of constant curvature $C$.

Generally, the cut locus Cut $(p)$ of $p$ on a closed surface $M$ is a local tree which may have infinitely many edges $[\mathbf{M 1}$, M2, GS]. So, the Hausdorff 1 -measure is used to measure a subset of Cut $(p)$. It is known that every compact subset of Cut $(p)$ of $p$ on a complete surface $M$ has finite total length (Hausdorff 1-measure) [He2, I]. As an application of our isoperimetric inequality, we derive an inequality involving the total length of the cut locus of a point on a closed surface.

\section{Sectors on a Surface.}

Definition 2.1. Let $A$ be a subset of $M$ and $p, q \in A$. The distance between $p$ and $q$ in $A$ is defined by

$$
d_{A}(p, q)=\inf _{c \in \Omega_{p, q}^{A}} \int_{c} d s,
$$

where $\Omega_{p, q}^{A}$ is the set of all piecewise smooth curve segments contained in $A$ joining $p$ and $q$. Let $B \subset M$ such that $A \cap B \neq \emptyset$. Then $d_{A}(p, B)=$ $\inf _{q \in A \cap B} d_{A}(p, q)$ denotes the distance from $p$ to $B$ in $A$.

Note that $d_{A}(\cdot, \cdot) \geq d_{M}(\cdot, \cdot)$ for any set $A \subset M$.

Definition 2.2. Let $A$ be a compact subset of $M$ and $G$ a subset of the boundary $\partial A$ of $A$. For $t \geq 0$, the parallel $G_{t}$ of $G$ in the distance $t$ in $A$ is defined by

$$
G_{t}=\left\{q \in A: d_{A}(q, G)=t\right\} .
$$

For a proper sector $D(\Gamma)$ in $M$, the parallel $\Gamma_{t}$ of $\Gamma$ in $D(\Gamma)$ is a piecewise smooth simple curve for small $t>0$. As $t$ gets larger, $\Gamma_{t}$ can have several components.

Relative Cut Locus. Let $D(\Gamma)$ be the proper sector with two geodesics $\gamma_{1}, \gamma_{2}$ on $M$ and a piecewise smooth simple curve segment $\Gamma:[a, b] \rightarrow M$ with corners $\Gamma\left(s_{i}\right)=x_{i}$ at $a=s_{1}<s_{2}<\cdots<s_{n+1}=b$. Let $N(s)$ be the inward unit normal vector field along $\Gamma$ with the right/left limits $N\left(s_{i}^{ \pm}\right)$at the corners $x_{i}$ of $\Gamma, i=1, \ldots, n+1$. With notational conventions, $N\left(s_{1}^{-}\right)=-\gamma_{1}^{\prime}\left(r_{1}\right)$ and $N\left(s_{n+1}^{+}\right)=-\gamma_{2}^{\prime}\left(r_{2}\right)$, let $\mathcal{N}_{i}$ be the set of all inward 
unit tangent vectors in $T_{x_{i}} M$ between $N\left(s_{i}^{-}\right)$and $N\left(s_{i}^{+}\right)$. Let

$$
\mathcal{N}=\bigcup_{i=0}^{n+1} \mathcal{N}_{i}
$$

where $\mathcal{N}_{0}=\{N(s): s \in[a, b]\}$. For each $v \in \mathcal{N} \cap T_{q} M, q \in \Gamma$, let $\gamma_{v}:[0, r] \rightarrow M$ be a geodesic such that $\gamma_{v}(0)=q$ and $\gamma_{v}^{\prime}(0)=v$. The point $z=\gamma_{v}(t)$ where $\gamma_{v}$ stops minimizing the distance $d_{D(\Gamma)}\left(\gamma_{v}(t), \Gamma\right)$ is called the relative cut point of $v \in \mathcal{N}$ in $D(\Gamma)$. The set of all such relative cut points of $v \in \mathcal{N}$ in $D(\Gamma)$ is called the relative cut locus of $\Gamma$ in $D(\Gamma)$, denoted by $\mathcal{C}_{\text {rel }}(\Gamma)$. If the exterior angle of $\Gamma$ at a corner $x_{i}$ is positive, then for all $v \in \mathcal{N}_{i}$, the relative cut point of $v$ in $D(\Gamma)$ is $x_{i}$ itself. Note also that $\mathcal{C}_{\text {rel }}(\Gamma)$ need not be a connected set.

Geodesic Sectors. For a point $p \in M$, let $\mathcal{U}$ be a (simply connected) normal neighborhood of $p$, and take polar coordinates $(r, \theta)$ on $\mathcal{U} \backslash\{p\}$ such that the metric can be written as

$$
d s^{2}=d r^{2}+f^{2} d \theta^{2}
$$

where $f=f(r, \theta)$ is the positive-valued function satisfying the initial conditions

$$
\lim _{r \rightarrow 0} f(r, \theta)=0, \quad \lim _{r \rightarrow 0} \frac{\partial f}{\partial r}(r, \theta)=1 .
$$

Let $\gamma_{1}, \gamma_{2}:[0, r] \rightarrow M, i=1,2$, be two geodesics starting at $p$ with the angle $0<\theta \leq \pi$ and $\beta:[0, \theta] \rightarrow \mathcal{U} \subset M$ a geodesic circular arc given by $\beta(s)=(r, s)$ in $\mathcal{U}$. The proper sector enclosed by $\gamma_{1}, \gamma_{2}$ and $\beta$ is called a geodesic sector denoted by $S_{r, \theta}$. We will call $\beta$ the circular boundary of $S_{r, \theta}$. The area $A_{r, \theta}$ and the arc length $L_{r, \theta}$ of the circular boundary of $S_{r, \theta}$ are respectively given by

$$
A_{r, \theta}=\int_{0}^{\theta} \int_{0}^{r} f(t, s) d t d s, \quad L_{r, \theta}=\int_{0}^{\theta} f(r, s) d s .
$$

Remark 2.3. A geodesic sector with vertex $p$ may cross the usual cut locus $\operatorname{Cut}(p)$ of $p$. One can easily construct a geodesic sector $S_{r, \theta}$ crossing the cut locus $\operatorname{Cut}(p)$ of $p$ on the cylinder $\mathbb{R} \times \mathbb{S}^{1}$.

Let $S_{r, \theta}^{C}$ denote a geodesic sector of radius $r$ and angle $\theta$ on a surface $M_{C}$ of constant curvature $K \equiv C$. Let $A_{r, \theta}^{C}$ and $L_{r, \theta}^{C}$ denote its area and the arc length of the circular boundary, respectively. The explicit expressions are:

$$
\begin{array}{cccc}
C: & a^{2}>0 & 0 & -a^{2}<0 \\
A_{r, \theta}^{C}: & 2 \theta \frac{\sin ^{2} \frac{a r}{2}}{a^{2}} & \frac{1}{2} \theta r^{2} & 2 \theta \frac{\sinh ^{2} \frac{a r}{2}}{a^{2}} \\
L_{r, \theta}^{C}: & \theta \frac{\sin a r}{a} & \theta r & \theta \frac{\sinh a r}{a} .
\end{array}
$$


One can easily verify the following formula:

$$
\left(L_{r, \theta}^{C}\right)^{2}=2 \theta A_{r, \theta}^{C}-C\left(A_{r, \theta}^{C}\right)^{2} .
$$

For the geodesic sectors on a surface with curvature $K \leq C$, we may have the following lemmas as immediate consequences of the formulas (2.1) and Lemma 7 in $[\mathbf{O s}]$ :

Lemma 2.4. Let $M$ be a surface with curvature $K \leq C$. Let $S_{r, \theta}$ be a geodesic sector on $M$ of radius $r$ and angle $\theta$. Then

$$
A_{r, \theta} \geq A_{r, \theta}^{C}
$$

with equality if and only if $S_{r, \theta}$ is isometric to $S_{r, \theta}^{C}$ on a surface $M_{C}$ of constant curvature $C$.

Lemma 2.5. Under the same assumptions as in Lemma 2.4, we have

$$
L_{r, \theta} \geq L_{r, \theta}^{C} .
$$

If $L_{\xi, \theta}=L_{\xi, \theta}^{C}$ for all $\xi \in(0, r]$, then $S_{r, \theta}$ is isometric to $S_{r, \theta}^{C}$ on a surface $M_{C}$ of constant curvature $C$.

\section{Isoperimetric Inequalities for Sectors on a Surface.}

Let $\beta:[a, b] \rightarrow M$ be a unit speed simple curve, and let $\mathbf{n}$ be a unit normal vector field along $\beta$. Then one can find a variation $\mathcal{X}:[a, b] \times(-\delta, \delta) \rightarrow M$ of $\beta$ given by

$$
\mathcal{X}(s, \xi)=\exp _{\beta(s)} \xi \mathbf{n}(s)
$$

for some $\delta>0$.

Let $L_{\mathcal{X}}(\xi)$ denote the arc length of the curve $\beta_{\xi}(s)=\mathcal{X}(s, \xi)$, which will be called a geodesic parallel of $\beta$. Then, from the first variation formula

$$
L_{\mathcal{X}}^{\prime}(0)=\int_{a}^{b} g\left(\beta^{\prime}, \nabla_{\beta^{\prime}} \mathbf{n}\right) d s=-\int_{a}^{b} \kappa d s
$$

where $\nabla$ is the Levi-Civita connection of $M$, we have

$$
L_{\mathcal{X}}(\xi)=L_{\mathcal{X}}(0)-\xi \int_{\beta} \kappa d s+o(\xi)
$$

where $\kappa$ is the geodesic curvature of $\beta$.

For the computation of the length of the parallel $\Gamma_{t}$ of $\Gamma$, we write $\Gamma=$ $\sum_{i=1}^{n} \beta^{i}$ where $\beta^{i}(i=1, \ldots, n)$ are smooth curves with inward unit normal $N$. For $t>0$ small, the parallel $\Gamma_{t}$ of $\Gamma$ in $D(\Gamma)$ consists of parts of the geodesic parallels $\beta_{t}^{i}$ of $\beta^{i}$ in $D(\Gamma)$ together with the geodesic circular arcs of radius $t$. Let

$$
t_{*}=\sup _{p \in \Gamma, q \in \mathcal{C}_{\mathrm{rel}}(\Gamma)} d_{D(\Gamma)}(p, q)
$$


and for $0 \leq t \leq t_{*}$, let

$$
D\left(\Gamma_{t}\right)=\left\{q \in D(\Gamma): d_{D(\Gamma)}(q, \Gamma) \geq t\right\} .
$$

Notice that $D\left(\Gamma_{t}\right)$ may not be a proper sector since it is not a connected set in general. We will denote the arc length of $\Gamma_{t}$ by $L(t)$ and the area of $D\left(\Gamma_{t}\right)$ by $A(t)$ as functions of $t$.

Lemma 3.1. Let $M$ be a surface with curvature $K \leq C$. Let $D(\Gamma)$ be a proper sector on $M$ with interior angle $\theta_{0} \leq \pi$ at the vertex. Then

$$
L^{\prime}(0) \leq C A(0)-\theta_{0} .
$$

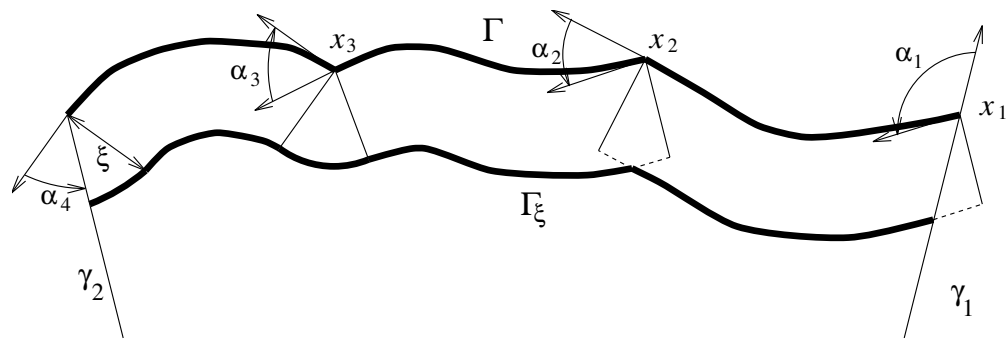

Figure 1.

Proof. Let $x_{i}(i=1,2, \ldots, n+1)$ be the corners of $\Gamma$ including end points and let $\alpha_{i}$ denote the exterior angle of $\Gamma^{*}$ at the corner $x_{i}$ (See Figure 1). We may assume that $\alpha_{i} \neq \pi$. Let $\mathcal{S}=\{2, \ldots, n\}$, and let $\mathcal{A}=\left\{i \in \mathcal{S}: \alpha_{i} \leq 0\right\}$ and $\mathcal{B}=\left\{j \in \mathcal{S}: \alpha_{j}>0\right\}$. For sufficiently small $\xi>0$, using the linear approximations for dotted parts of $\beta_{\xi}^{i}$ (say, at $x_{2}$ in Figure 1), we have, with the help of (3.2),

$$
\begin{aligned}
L(\xi)= & L(0)-\xi \int_{\Gamma} \kappa d s-\sum_{i \in \mathcal{A}} \xi \alpha_{i}-\sum_{j \in \mathcal{B}} 2 \xi \tan \left(\alpha_{j} / 2\right) \\
& -\xi \widetilde{\alpha}_{1}-\xi \widetilde{\alpha}_{n+1}+o(\xi),
\end{aligned}
$$

where $\kappa$ is the geodesic curvature of $\Gamma$ and

$$
\widetilde{\alpha}_{k}= \begin{cases}\alpha_{k}-\pi / 2 & \text { if } \alpha_{k} \leq \pi / 2 \\ \tan \left(\alpha_{k}-\pi / 2\right) & \text { if } \alpha_{k}>\pi / 2 .\end{cases}
$$

Using that $\tan \alpha \geq \alpha$ for $\alpha \geq 0$ and $\widetilde{\alpha}_{k} \geq \alpha_{k}-\pi / 2$, we have

$$
\begin{aligned}
L^{\prime}(0) & =-\int_{\Gamma} \kappa d s-\sum_{i \in \mathcal{A}} \alpha_{i}-\sum_{j \in \mathcal{B}} 2 \tan \left(\alpha_{j} / 2\right)-\widetilde{\alpha}_{1}-\widetilde{\alpha}_{n+1} \\
& \leq-\int_{\Gamma} \kappa d s-\sum_{i=1}^{n+1} \alpha_{i}+\pi
\end{aligned}
$$


Since $\gamma_{1}, \gamma_{2}$ are geodesics, by the Gauss-Bonnet formula,

$$
L^{\prime}(0) \leq \int_{D(\Gamma)} K d A-\theta_{0} .
$$

Finally, we have

$$
L^{\prime}(0) \leq C A(0)-\theta_{0}
$$

from the curvature condition that $K \leq C$.

Note that, for sufficiently small $t>0, \Gamma_{t}$ has one component of a piecewise smooth non-closed simple curve segment. Computation as in Lemma 3.1 thus gives that

$$
L^{\prime}(t) \leq C A(t)-\theta_{0}
$$

for sufficiently small $t>0$.

Note also that $\Gamma_{t}$ could be the union of at most finite number of piecewise smooth non-closed curves, piecewise smooth closed curves and points in general. For a piecewise smooth closed curve, by the same way as in Lemma 3.1 we have the following:

Lemma 3.2. Let $M$ be a surface with curvature $K \leq C$ and $D$ a nondegenerate compact subset of $M$ with the boundary $\partial D=G$ which is a piecewise smooth simple curve. Let $G_{\xi}$ denote the parallel of $G=\partial D$ in $D$ and $\ell(t)=L\left(G_{t}\right)$. Then

$$
\ell^{\prime}(0) \leq C A(D)-2 \pi
$$

The following facts come from the results of [Fi, pp. 303-332] by a slight modification (also see [CF, p. 86]): $L(t)$ is continuous for all but at most a finite number of $t$ in $\left[0, t_{*}\right]$ at which $L(t)$ has a jump discontinuity, however $A(t)$ is continuous; $A^{\prime}(t)=-L(t)$ for almost all $t \in\left[0, t_{*}\right]$ (cf. [Ha, p. 706]).

Theorem 3.3. Let $M$ be a surface with curvature $K \leq C$. Let $D(\Gamma)$ be a proper sector on $M$ with interior angle $\theta_{0} \leq \pi$ at the vertex. Then

$$
L^{\prime}(t) \leq C A(t)-\theta_{0}
$$

for almost all $t \in\left[0, t_{*}\right]$.

Proof. Let $n_{t}$ and $m_{t}$ be the numbers of components of piecewise smooth non-closed curves $\Gamma_{t}^{i}$ and piecewise smooth closed curves $\Omega_{t}^{j}$ of $\Gamma_{t}$, respectively. For almost all $t \in\left[0, t_{*}\right]$, we may write

$$
\Gamma_{t}=\sum_{i=1}^{n_{t}} \Gamma_{t}^{i}+\sum_{j=1}^{m_{t}} \Omega_{t}^{j} .
$$

Note that each end point of $\Gamma_{t}^{i}$ is either on $\gamma_{1}$ or on $\gamma_{2}$ so that $\Gamma_{t}^{i}$ and $\gamma_{1}$ and/or $\gamma_{2}$ bound a simply connected compact set, denoted by $D\left(\Gamma_{t}^{i}\right)$. Each 
$\Omega_{t}^{j}$ itself also bounds simply connected compact set, denoted by $D\left(\Omega_{t}^{j}\right)$. Thus we may write

$$
D\left(\Gamma_{t}\right)=\left(\bigcup_{i=1}^{n_{t}} D\left(\Gamma_{t}^{i}\right)\right) \bigcup\left(\bigcup_{j=1}^{m_{t}} D\left(\Omega_{t}^{j}\right)\right) .
$$

For the sake of brevity, we use the notations: $L_{i}(t)=L\left(\Gamma_{t}^{i}\right), A_{i}(t)=$ $A\left(D\left(\Gamma_{t}^{i}\right)\right), \ell_{j}(t)=L\left(\Omega_{t}^{j}\right)$ and $B_{j}(t)=A\left(D\left(\Omega_{t}^{j}\right)\right)$. Then by the same computation as (3.3) we have

$$
L_{i}^{\prime}(t) \leq C A_{i}(t)-\theta_{i},
$$

where $\theta_{0} \leq \theta_{i} \leq \pi$. By Lemma 3.2,

$$
\ell_{j}^{\prime}(t) \leq C B_{j}(t)-2 \pi
$$

Thus,

$$
\begin{aligned}
L^{\prime}(t) & =\sum_{i}^{n_{t}} L_{i}^{\prime}(t)+\sum_{j}^{m_{t}} \ell_{j}^{\prime}(t) \\
& \leq \sum_{i}^{n_{t}}\left(C A_{i}(t)-\theta_{i}\right)+\sum_{j}^{m_{t}}\left(C B_{j}(t)-2 \pi\right) \\
& \leq C A(t)-\theta_{0}
\end{aligned}
$$

for almost all $t \in\left[0, t_{*}\right]$.

Theorem 3.4. Let $M$ be a surface with curvature $K \leq C$. Let $D(\Gamma)$ be a proper sector on $M$ with interior angle $\theta_{0} \leq \pi$ at the vertex. Then

$$
L^{2}(s)-L^{2}(t) \geq 2 \theta_{0}(A(s)-A(t))-C\left(A^{2}(s)-A^{2}(t)\right)
$$

for $s<t \in\left[0, t_{*}\right]$.

Proof. By multiplying $A^{\prime}(t)=-L(t) \leq 0$ to the inequality (3.4), we get

$$
-L(t) L^{\prime}(t) \geq C A(t) A^{\prime}(t)-\theta_{0} A^{\prime}(t)
$$

for almost all $t \in\left[0, t_{*}\right]$. Note that $L(t)$ is continuous on $\left[0, t_{*}\right]$ except for a finite number of points $0<t_{1}<t_{2}<\cdots<t_{m}<t_{*}$. Let $I_{j}=\left[t_{j-1}, t_{j}\right]$, $j=1,2, \ldots, m+1$, where $t_{0}=0, t_{m+1}=t_{*}$. For $s<t$ in $\left[0, t_{*}\right]$, we may assume that $s \in I_{i}$ and $t \in I_{j}$ for some $i \leq j$. By direct computation, we have

$$
-\int_{s}^{t} L(t) L^{\prime}(t) d t=\frac{1}{2}\left(L^{2}(s)-L^{2}(t)\right)+\frac{1}{2} \sum_{k=i}^{j-1}\left(L^{2}\left(t_{k}^{+}\right)-L^{2}\left(t_{k}^{-}\right)\right),
$$


where $h\left(r^{ \pm}\right)$, as usual, stands for the right/left limits of a function $h$ at $r$. Notice that $L\left(t_{k}^{+}\right)<L\left(t_{k}^{-}\right)$and $A\left(t_{k}^{+}\right)=A\left(t_{k}^{-}\right)$. Thus we have

$$
-\int_{s}^{t} L(t) L^{\prime}(t) d t \leq \frac{1}{2}\left(L^{2}(s)-L^{2}(t)\right)
$$

Similarly,

$$
\begin{aligned}
\int_{s}^{t} A(t) A^{\prime}(t) d t & =-\frac{1}{2}\left(A^{2}(s)-A^{2}(t)\right), \\
-\int_{s}^{t} A^{\prime}(t) d t & =A(s)-A(t) .
\end{aligned}
$$

Therefore, from (3.9)-(3.12), we have

$$
\begin{aligned}
L^{2}(s)-L^{2}(t) & \geq-2 \int_{s}^{t} L(t) L^{\prime}(t) d t \\
& \geq 2 C \int_{s}^{t} A(t) A^{\prime}(t) d t-2 \theta_{0} \int_{s}^{t} A^{\prime}(t) d t \\
& =2 \theta_{0}(A(s)-A(t))-C\left(A^{2}(s)-A^{2}(t)\right) .
\end{aligned}
$$

We are now ready to state and prove our main result.

Theorem 3.5. Let $M$ be a surface with curvature $K \leq C$. Let $D(\Gamma)$ be a proper sector on $M$ with interior angle $\theta_{0} \leq \pi$ at the vertex. Then

$$
L^{2}(\Gamma) \geq 2 \theta_{0} A(D(\Gamma))-C A^{2}(D(\Gamma)),
$$

where equality holds only when $D(\Gamma)$ is isometric to a geodesic sector on a surface $M_{C}$ of constant curvature $K \equiv C$.

Proof. By setting $t \rightarrow t_{*}$ in (3.8) of Theorem 3.4, we get

$$
L^{2}(s) \geq 2 \theta_{0} A(s)-C A^{2}(s) .
$$

Now at $s=0$, we get the inequality (3.13).

For a geodesic sector on a surface $M_{C}$ of constant curvature $K \equiv C$, it is quite clear that the equality holds in (3.13) by (2.3).

Suppose now that the equality $L^{2}(0)=2 \theta_{0} A(0)-C A^{2}(0)$ holds. Then from (3.8) with $s=0$ together with (3.14) we get

$$
L^{2}(t)=2 \theta_{0} A(t)-C A^{2}(t),
$$

for all $0 \leq t \leq t_{*}$. Since $\Gamma_{t_{*}}$ is in the relative cut locus $\mathcal{C}_{\text {rel }}(\Gamma)$ of $\Gamma$ in $D(\Gamma)$, $D\left(\Gamma_{t_{*}}\right)$ is contained in $\mathcal{C}_{\text {rel }}(\Gamma)$. That is, $A\left(t_{*}\right)=0$ and so by $(3.15) L\left(t_{*}\right)=0$. By differentiation,

$$
L^{\prime}(t)=-\theta_{0}+C A(t)
$$


for all $0 \leq t \leq t_{*}$. Therefore, equalities hold for all $0 \leq t \leq t_{*}$ in inequalities in the proof of Theorem 3.3. This implies that, for $t<t_{*}$, the exterior angles at the end points of $\Gamma_{t}$ are less than or equal to $\pi / 2$ and there are no corners (which are not end points) on $\Gamma_{t}$ at which the exterior angle of $\Gamma_{t}^{*}$ is positive. In addition, for each $v \in T_{q} M \cap \mathcal{N}$, which is the set defined as in Section 2, the geodesic $\gamma_{v}:\left[0, t_{*}\right] \rightarrow M$ such that $\gamma_{v}(0)=q \in \Gamma, \gamma_{v}^{\prime}(0)=v$ satisfies $d_{D(\Gamma)}\left(\gamma_{v}(t), q\right)=t$ and $\gamma_{v}(t) \in \Gamma_{t}$ for each $t \in\left[0, t_{*}\right]$. That is, $\mathcal{C}_{\text {rel }}(\Gamma) \subseteq \Gamma_{t_{*}}$. Therefore, $\mathcal{C}_{\text {rel }}(\Gamma)=\Gamma_{t_{*}}$ is the set of a single point, say $\{p\}$. Moreover, no geodesics starting at $p$ intersect before the distance $t_{*}$ in $D(\Gamma)$, so $D(\Gamma)$ is a geodesic sector of radius $t_{*}$ and angle $\theta_{0}$ centered at $p$. By (3.15) and (3.16), $L:\left[0, t_{*}\right] \rightarrow \mathbb{R}$ satisfies the following ODE

$$
L^{\prime \prime}(t)=-C L(t), \quad L\left(t_{*}\right)=0, \quad L^{\prime}\left(t_{*}\right)=-\theta_{0} .
$$

By comparing the solution of (3.17) with $L_{t, \theta}^{C}$ in (2.2) for a geodesic sector on $M_{C}$, one can see that $D(\Gamma)$ is isometric to $S_{t_{*}, \theta_{0}}^{C}$ by Lemma 2.5 .

If $M=\mathbb{E}^{2}$ and we set $C=0$, then Theorem 3.5 implies the result of C. Bandle [Ba1, Ba2]. Similar isoperimetric inequalities on Lorentzian surfaces were obtained by the authors $[\mathbf{B H}, \mathbf{B}]$.

Remark 3.6. The condition that $\theta_{0} \leq \pi$ in Theorem 3.5 is essential. One can construct a proper sector for which the isoperimetric inequality (3.13) does not hold in the following way: In $\mathbb{E}^{2}$, consider a proper sector with $\Gamma=\Gamma^{1} \cup \Gamma^{2}$, where $\Gamma^{1}$ is a semi-circle of radius $r$ centered at $q$ and $\Gamma^{2}$ is a circular arc of angle $0<\varphi<\pi$ and radius $\alpha r(0<\alpha<1)$ centered at $p$ (see Figure 2). Take $C=0$, then

$$
L^{2}(\Gamma)=(\pi+\varphi \alpha)^{2} r^{2}<(\pi+\varphi)\left(\pi+\varphi \alpha^{2}\right) r^{2}=2 \theta_{0} A(D(\Gamma)) .
$$

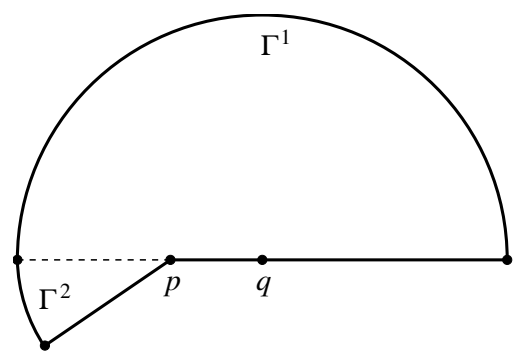

Figure 2. 
Remark 3.7. The isoperimetric inequality (3.13) holds for a closed, simply connected, bounded set $D$ having the boundary $\Gamma^{*}=\gamma_{1} \cup \Gamma \cup \gamma_{2}$, where $\gamma_{1}(0)=\gamma_{2}(0), \gamma_{1}\left(r_{1}\right)=\gamma_{2}\left(r_{2}\right)=\Gamma(a)=\Gamma(b)$ by approximating $\Gamma^{*}$ by $\Gamma_{\varepsilon}^{*}$, where $\Gamma_{\varepsilon}^{*}$ is a closed curve obtained from $\Gamma^{*}$ by changing the parts of $\Gamma^{*}$ contained in a geodesic ball of radius $\varepsilon$ at $\gamma_{1}\left(r_{1}\right)=\gamma_{2}\left(r_{2}\right)$ so that $\Gamma_{\varepsilon}^{*}$ is piecewise smooth and simple.

We now consider a special case of a proper sector: Suppose that $\gamma_{i}$ : $\left[0, r_{i}\right] \rightarrow M(i=1,2)$ are two geodesic segments such that $\gamma_{1}(0)=\gamma_{2}(0)=p$, $\gamma_{1}\left(r_{1}\right)=\gamma_{2}\left(r_{2}\right)=q$ and $\gamma_{1}\left(\left(0, r_{1}\right)\right) \cap \gamma_{2}\left(\left(0, r_{2}\right)\right)=\emptyset$. Such a sector will be called an oval sector. Notice that there are no such oval sectors on a surface with curvature $K \leq 0$. Let $\theta_{1}, \theta_{2}$ be the interior angles of $D$ at $p, q$, respectively. From Theorem 3.5, we have:

Corollary 3.8. Let $M$ be a surface with curvature $K \leq C$ for a positive constant $C$. Let $D$ be an oval sector enclosed by $\gamma_{1}, \gamma_{2}$ with $\theta_{1}, \theta_{2} \leq \pi$ on M. Then

$$
A(D) \geq \frac{2 \theta_{*}}{C}
$$

where $\theta_{*}=\max \left\{\theta_{0}, \theta_{1}\right\}$. Equality holds only when $D$ is isometric to a geodesic sector of radius $\pi / \sqrt{C}$ and angle $\theta_{1}=\theta_{2}$ on a surface of constant curvature $K \equiv C$.

The equality case of Corollary 3.8 is based on the following fact: For an oval sector $D$ with $\theta_{1}, \theta_{2} \leq \pi$ on a surface $M$ with curvature $K \leq C$, by the Gauss-Bonnet formula, one can obtain the inequality

$$
A(D) \geq \frac{1}{C}\left(\theta_{0}+\theta_{1}\right)
$$

where equality holds only when $M$ is a surface of constant curvature $C$.

The following is an immediate consequence of Corollary 3.8:

Corollary 3.9. Let $M$ be a surface with curvature $K \leq C$ for a positive constant $C$. Suppose that $\gamma_{1}, \gamma_{2}$ are two geodesics starting at a point $p \in M$ with angle $\theta$ at $p$ and $D$ is a simply connected domain on $M$ containing $\gamma_{1}, \gamma_{2}$ with area less than $\frac{2 \theta}{C}$. Then $\gamma_{1}$ and $\gamma_{2}$ never meet again in $D$.

\section{Lengths of the cut locus.}

Let $M$ be a closed surface (i.e., a compact surface without boundary) with area $A(M)$. For $v \in T_{p} M, p \in M$, denote by $\gamma_{v}$ the unique geodesic satisfying $\gamma_{v}^{\prime}(0)=v$. Define $\rho(v)=\sup \left\{t \in \mathbb{R}: \gamma_{v}\right.$ is minimal on $\left.[0, t]\right\}$. Then $\rho(v)$ is continuous on the set $\mathbb{S}_{p}=\left\{v \in T_{p} M:\|v\|=1\right\}$ and the values of $\rho$ on $\mathbb{S}_{p}$ are bounded (by the diameter of $M$ ). Note that, if $w=\lambda v \in T_{p} M$ $(\lambda \geq 0)$, then $\rho(v)=\lambda \rho(w)$. Let

$$
U_{p}=\left\{v \in T_{p} M: \rho(v)>1\right\} .
$$


Then $U_{p}$ is a bounded set in $T_{p} M$ and the (usual) cut locus of $p$ is

$$
\operatorname{Cut}(p)=\exp _{p}\left(\partial U_{p}\right) \text {. }
$$

It is well known that, for $p \in M$,

$$
M=\mathcal{U}_{p} \dot{\cup} \operatorname{Cut}(p),
$$

where $\mathcal{U}_{p}=\exp _{p}\left(U_{p}\right)$. Note that $\operatorname{Cut}(p)$ is a deformation retract of $M \backslash\{p\}$. Hence, on any orientable closed surface $M$ of genus $g$, Cut $(p)$ of any point $p \in M$ contains $2 g$ closed curves, which form a set of generators for the fundamental group of $M$. It is known that any compact subset of $\operatorname{Cut}(p)$ of $p$ on a closed surface $M$ has finite Hausdorff 1-measure (cf. $[\mathbf{H e 2}, \mathbf{I}]$ ). Thus any path in $\operatorname{Cut}(p)$ is rectifiable ([He1, Proposition 5.1]) and the Hausdorff 1 -measure of a path in $\operatorname{Cut}(p)$ is its arc length ([Fa, p. 29]). Using our isoperimetric inequality (4.1) in Theorem 4.2, we will derive an inequality involving the Hausdorff 1-measure of the cut locus of a point in a closed orientable surface.

Lemma 4.1. Let $M$ be a closed surface and $p \in M$. Then there is a geodesic segment through $p$ such that its end points are in $\operatorname{Cut}(p)$ and it bisects $\mathcal{U}_{p}$ in area.

Proof. For a unit vector $v \in T_{p} M$, denote $c_{v}:[-\rho(-v), \rho(v)] \rightarrow M$ the unique geodesic segment such that $c_{v}(s)=\gamma_{-v}(-s)$ for $s \in[-\rho(-v), 0]$ and $c_{v}(t)=\gamma_{v}(t)$ for $t \in[0, \rho(v)]$. Then $c_{v}(-\rho(-v))=\gamma_{-v}(\rho(-v)), c_{v}(\rho(v))=$ $\gamma_{v}(\rho(-v))$ are in $\operatorname{Cut}(p)$ of $p$ and $c_{v}(0)=p$. Clearly, for each $v \in \mathbb{S}_{p}, c_{v}$ splits $\mathcal{U}_{p}$ into two pieces. We take one piece of these for each $v$ continuously and name it $D_{v}$. Let $A(v)$ be the area of $D_{v}$. Then $A(v)$ is a continuous function on $\mathbb{S}_{p}$ since $\rho$ is continuous on $\mathbb{S}_{p}$. By the mean value theorem, there is a $v_{0} \in \mathbb{S}_{p}$ such that $A\left(v_{0}\right)=\frac{1}{2} A(M)$, and $c_{v_{0}}$ is a desired one.

Theorem 4.2. Let $M$ be a closed surface with curvature $K \leq C$ and $\ell$ the total length (the Hausdorff 1-measure) of the cut locus $\operatorname{Cut}(p)$ of $p \in M$. Then

$$
\ell^{2} \geq \pi A(M)-\frac{C}{4} A^{2}(M)
$$

Proof. Let $\gamma:[a, b] \rightarrow M$ be a geodesic segment bisecting $U_{p}$ into $D_{1}, D_{2}$ with $A\left(D_{1}\right)=A\left(D_{2}\right)$ as in Lemma 4.1. Then there is a (continuous) path $\widetilde{\Gamma}$ in $\operatorname{Cut}(p)$ joining $\gamma(a)$ and $\gamma(b)$ so that $\gamma$ and $\widetilde{\Gamma}$ constitute the common boundary of $D_{1}$ and $D_{2}$. As the path $\widetilde{\Gamma}$ may not be piecewise smooth and $\widetilde{\Gamma}$ is compact, for any $\varepsilon>0$ we choose a piecewise simple curve segment $\Gamma$ joining $\gamma(a)$ and $\gamma(b)$ such that $\Gamma$ is contained in the $\varepsilon$-neighborhood of $\widetilde{\Gamma}$ and $L(\Gamma) \leq L(\widetilde{\Gamma})$. Let $D_{1}^{\prime}, D_{2}^{\prime}$ be two domains with boundary $\gamma$ and $\Gamma$ corresponding to $D_{1}, D_{2}$, respectively. By Theorem 3.5 and Remark 3.7,

$$
L^{2}(\Gamma) \geq 2 \pi A\left(D_{1}^{\prime}\right)-C A^{2}\left(D_{1}^{\prime}\right), \quad L^{2}(\Gamma) \geq 2 \pi A\left(D_{2}^{\prime}\right)-C A^{2}\left(D_{2}^{\prime}\right) .
$$


Combining these with the fact that $A\left(D_{i}^{\prime}\right)=A\left(D_{i}\right)+O\left(\varepsilon^{2}\right)$ for $i=1,2$,

$$
L^{2}(\widetilde{\Gamma}) \geq 2 \pi A\left(D_{1}\right)-C A^{2}\left(D_{1}\right), \quad L^{2}(\widetilde{\Gamma}) \geq 2 \pi A\left(D_{2}\right)-C A^{2}\left(D_{2}\right) .
$$

Since $\widetilde{\Gamma} \subset \operatorname{Cut}(p)$ and $L(\widetilde{\Gamma})$ is equal to the Hausdorff 1-measure of $\widetilde{\Gamma}$, which is less than or equal to the total length $\ell$ of $\operatorname{Cut}(p)$, we get

$$
\ell^{2} \geq \pi A(M)-\frac{C}{4} A^{2}(M)
$$

Example 4.3. Let $T^{2}$ be the flat torus obtained by identifying the opposite sides of quadrilateral ABCD (see Figure 3). Then the cut locus $\operatorname{Cut}(p)$ of middle point $p \in T^{2}$ is the set formed by the line segments $\mathrm{AB}$ and $\mathrm{BC}$, and so $\ell=a+b$, where $a$ and $b$ are the length of the line segments $\mathrm{AB}$ and $\mathrm{BC}$, respectively. The area of $T^{2}$ is $a b$. Take $C=0$ as usual, then (4.1) gives a well-known inequality

$$
(a+b)^{2} \geq \pi a b
$$

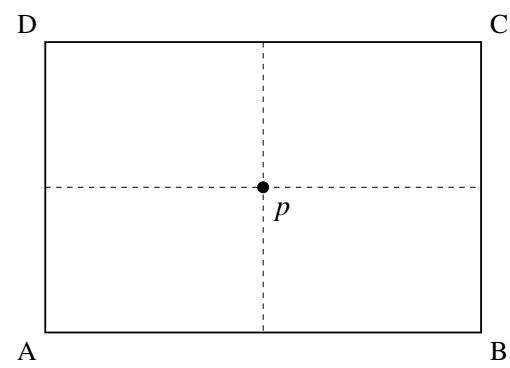

Figure 3.

Example 4.4. Let $P^{2}(\mathbb{R})$ be the projective plane of constant curvature $C=1$, obtained by identifying the antipodal points of the unit sphere $\mathbb{S}^{2}$. The cut locus $\operatorname{Cut}(p)$ of a point $p$ of $P^{2}(\mathbb{R})$ is the set obtained by identifying the antipodal points of the equator of $p$. Since $\ell=\pi$ and $A\left(P^{2}(\mathbb{R})\right)=2 \pi$, we get equality in (4.1). On $\mathbb{S}^{2}$, trivially we also get equality.

It is well known that any closed orientable surface of genus $g>1$ carries a metric with constant negative curvature [GHL, p. 167], and that the area of a closed orientable surface of genus $g>1$ and curvature -1 is $4 \pi(g-1)$ [GHL, p. 169]. Combining these and Theorem 4.2 we have: 
Corollary 4.5. Let $M$ be a closed orientable surface of genus $g>1$ with curvature -1 . Then

$$
\ell^{2} \geq 4 \pi^{2} g(g-1)
$$

Acknowledgement. H. Bahn would like to thank Professor Paul Ehrlich for helpful conversations while he was a visitor to the University of Florida.

\section{References}

[Al] A.D. Alexandroff, Isoperimetric inequalities for curved surfaces, Dokl. Akad. Nauk. SSSR, 47 (1945), 235-238.

[B] H. Bahn, Isoperimetric inequalities and conjugate points on Lorentzian surfaces, J. Geom., to appear.

$[\mathrm{BH}]$ H. Bahn and S. Hong, Isoperimetric inequalities for sectors on the Minkowski 2spacetime, J. Geom., 63 (1998), 17-24.

[Ba1] C. Bandle, A generalization of the method of interior parallels, and isoperimetric inequalities for membranes with partially free boundary, J. Math. Anal. Appl., 39 (1972), 166-176.

[Ba2] - Isoperimetric Inequalities and Applications, Pitman, London, 1980.

[BdC] J.L. Barbosa and M. do Carmo, A proof of a general isoperimetric inequality for surfaces, Math. Z., 162 (1978), 245-261.

[CF] I. Chavel and E.A. Feldman, Isoperimetric inequalities on curved surfaces, Adv. Math., 37 (1980), 83-98.

[Fa] K. Falconer, The Geometry of Fractal Sets, Cambridge Univ. Press, London, 1985.

[Fi] F. Fiala, Le problème des isopérimètres sur les surfaces ouverts à courbure positive, Comment. Math. Helv., 13 (1940-41), 293-396.

[GHL] S. Gallot, D. Hulin and J. Lafontaine, Riemannian Geometry (2/e), SpringerVerlag, Berlin, 1990.

[GS] H. Gluck and D. Singer, Scattering of geodesic fields I, Ann. of Math., 108(2) (1978), 347-372.

[Ha] P. Hartman, Geodesic parallel coordinates in the large, Amer. J. Math., 86 (1964), 705-727.

[He1] J.J. Hebda, Parallel translation of curvature along geodesics, Trans. Amer. Math. Soc., 299 (1987), 559-572.

[He2] _ Metric structure of cut loci in surfaces and Ambrose's problem, J. Differential Geom., 40 (1994), 621-642.

[I] J.-I. Itoh, The length of a cut locus on a surface and Ambrose's problem, J. Differential Geom., 43 (1996), 642-651.

[M1] S.B. Myers, Connections between differential geometry and topology I: Simply connected surfaces, Duke Math. J., 1 (1935), 376-391.

[M2] - Connections between differential geometry and topology II: Closed surfaces, Duke Math. J., 2 (1936), 95-102. 
[Os] R. Osserman, Bonnesen-style isoperimetric inequalities, Amer. Math. Monthly, 86 (1979), 1-29.

Received March 10, 1999 and revised June 23, 1999. This work was supported by POSTECH Research Fund and TGRC-KOSEF.

National Computerization Agency

YONGIN 449-717

KorEA

E-mail address: bahn@nca.or.kr

Department of Mathematics

Pohang University of Science And Technology

POHANG 790-784

KOREA

E-mail address: sungpyo@postech.ac.kr 